\title{
Evaluación de la estatura durante el desarrollo puberal
}

\author{
Dra, Ada Mauricei N. ${ }^{1}$; Dra. Raquel Burrows A. ${ }^{1}$; T.M. Laura Leiva B. ${ }^{1}$; \\ Sr. Abraham Zvaighaft F. ${ }^{1}$; Dr. Ariel Kauschansky ${ }^{2}$; Dr. Santiago Muzzo B. ${ }^{1}$ \\ Evaluation of height during puberal development
}

A cross-sectional study in 665 heatthy children of both sexes, aged 11 to 16 years (394 girls) was done to evaluate the adecuation of their height to chronologic age $(\mathrm{H} / \mathrm{A})$, and to puberal development $(\mathrm{H} / \mathrm{P})$ by means of Tanner's scales. (Breast stages in girls and pubic hair stages in boys according to Tanner, were considered). Subjects with early puberal development showed significantly higher $\mathrm{H} / \mathrm{A}$ than $\mathrm{H} / \mathrm{P}$ rates resulting in nomal height for age but low height for puberal stage. Children with late puberal development had lower $\mathrm{H} / \mathrm{A}$ than $\mathrm{H} / \mathrm{P}$ rates and their heights wete low for age but normal for puberal stage. In the evaluation of the height it is necessary to consider the at tained puberal stage, in order to more accurate prognosis of expected adult heights.

(Key words: Height. Chronologic age. Puberal development).

Para valorar el crecimiento y desarrollo se utilizan diversos parámetros ${ }^{1,2}$. La evaluación mediante parámetros antropométricos generalmente se hace de acuerdo a edad y sexo del examinado, siendo el crecimiento en longitud uno de los más utilizados en clínica. La evaluación del grado de maduración sexual del adolescente es importante, para estimar los distintos tipos de variaciones fisiológicas, formular pronósticos de estatura y diagnosticar alteraciones patológicas ${ }^{3-9}$

Se ha descrito, en adolescentes americanas una estatura crítica en el momento del inicio de la pubertad; que influye en la estatura final alcanZada $^{10,11}$. Frisch ${ }^{12}$ predice la estatura final de las nî̃as, considerando la estatura y la edad en el momento de la menarca. Se conoce que el pronóstico de la estatura final se efectúa mejor con la edad ósea o el grado de desarrollo puberal, que con la edad cronológica del examinado13-18. En sujetos que presentan los eventos puberales a una edad promedio, el pronóstico de estatura final obtenida según edad cronológica (T/E) sería similar a la pronosticada según desarrollo puberal (T/P). En casos de eventos puberales tempranos o tardíos ambos pronósticos difieren. En una pubertad normal se observa una aceleración de la

1. Unidad de Endocrinología, Instituto de Nutrición y Tecnología de los Alimentos, Universidad de Chile.

2. Hasharon Hospital, Peta Tiqua, Israel.

Este trabajo fue financiado en parte por el proyecto 93/8S de FONDECYT y presentado en las XI Jornadas Chilenas de Pediatría, realizadas desde el 28 al 30 de Noviembre de 1985, en Santiago. velocidad de crecimiento y aparición de los caracteres sexuales secundarios. La aceleración puberal del crecimiento se presenta en momentos determinados del đesarrollo puberal, más temprano en la niña que en el varón. La mayoría de las nifías alcanzan la máxima velocidad de crecimiento lineal (MVC) durante las etapas de desarrollo mamario y dej vello púbico 2 a 3 y la menarquia que se produce en etapa 4. También en la mayoría de las niñas, la menarca ocurre después de haber presentado la MVC. En los varones en cambio, la MVC muy raramente ocurre antes de la etapa 4. Esto tiene una importancia práctica para efectuar un pronóstico adecuado de talla final, especialmente en niños con estatura alterada para la edad, y con variaciones constitucionales de su inicio puberal que implican una talla final normal.

Nos interesó estudiar en escolares de Santiago, las diferencias que existen al evaluar la estatura de acuerdo a la edad cronológica o al desarrollo puberal alcanzado según presentaran eventos puberales tempranos, normales o tardíos.

\section{MATERIAL Y METODO}

Este estudio corresponde a una parte del proyecto sobre características de la pubertad del escolar chileno realizado por la Unidad de Endocrinología de INTA entre 1984 y 1985. Se efectuó un corte transversal de una muestra aleatoria representativa de 1879 escolares del Area Metropolitana de Santiago, 1.048 niñas de 11 a 14 años te edad y 831 varones de 13 a 16 años de diferentes niveles socioeconómicos.

De este estudio se seleccionaron 156 ninas de 11 años a 11 años 11 meses y 73 de 13 años a 13 años 11 meses en etapa II y IV de desarrollo mamario, y 154 varones de 13 años a 13 años 11 meses y 117 de 15 años 
a 15 años 11 meses en etapas II y IV de desarrollo de vello púbico.

El grado de desarrollo puberal y las adecuaciones estaturales según edad cronológica y puberal, se evaluaron de acuerdo a las tablas de Tanner ${ }^{2-B-9-20 . ~ L a ~}$ adecuación estatural se consideró $100 \%$ cuando correspondió al percentil 50 para la edad cronológica. Se consideró edad de desarrollo puberal aquella en la cual el $50 \%$ de la población estudiada por Tanner habría alcanzado el evento puberal analizado. El análisis estadístico de los resultados se realizó mediante prueba de Student pareada.

\section{RESULTADOS}

La Tabla 1 describe la estatura de escolares de ambos sexos a diferentes edades $y$ en etapas II $y$ IV de desarrollo puberal. El promedio estatural fue significativamente mayor en los escolares de mayor edad cronológica y en una misma etapa de desarrollo.

La Tabla 2 muestra los promedios de adecua. ción de estatura de mujeres de 11 años, en etapas
II y IV de desarrollo mamario, expresados según edad cronológica $(\mathrm{T} / \mathrm{E})$ y de desarrollo puberal (T/P). El porcentaje de adecuación de $T / E$ es menor en las escolares en etapa II, que en las en etapa IV; mientras que la $\mathrm{T} / \mathrm{P}$, es significativa. mente mayor en las adolescentes en etapa ll. En escolares en etapa II, no hubo diferencias entre ambas adecuaciones estaturales, en cambio en etapa IV la $T / P$ es significativamente menor que la $T / E$,

En la Tabla 3 se analiza la estatura de niñas de 13 atios, en etapas II y IV de desarrollo mamario. Nuevamente el porcentaje de adecuación de $T / E$ fue significativamente menor en escolares en etapa II que en IV en tanto que el porcentaje de adecuación de $T / P$ es menor en las escolares en etapa IV que en la II. Al comparar ambas adecuaciones estaturales, la $T / E$ es significativamente menor a la $\mathrm{T} / \mathrm{P}$ en ambos grupos, siendo esta diferencia mayor en escolares en etapa II.

La Tabla 4 muestra la adecuación estatural de

Tabla 1.

Estatura en escolares de diferentes edades según grado de desarrollo puberal (Tanner)

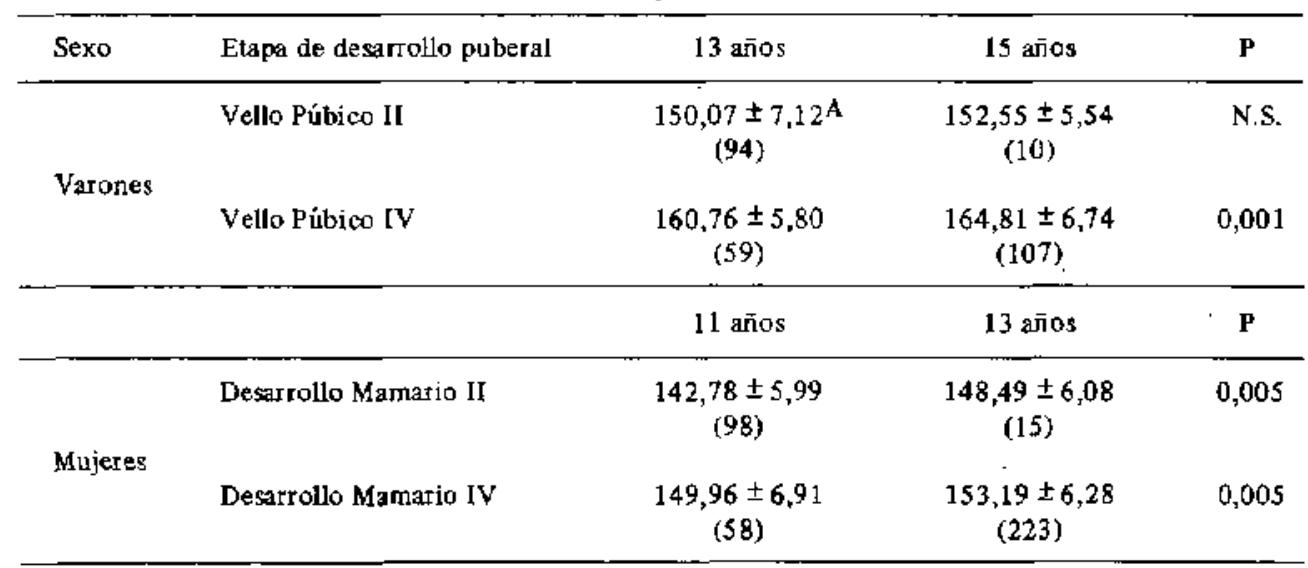

$\underline{A}=\overrightarrow{\mathrm{X}} \pm \mathrm{DE}$

(N)

Tabla 2.

Taila en escolares de 11 años de sexo femenino con diferentes etapas de desarrollo mamario (Tanner)

\begin{tabular}{lcccc}
\hline & II & IV & P \\
\hline \%Talla: & Según edad cronológica & $\begin{array}{c}98,55 \pm 4,36 \mathrm{~A} \\
(98)\end{array}$ & $\begin{array}{c}103,23 \pm 4,89 \\
(56)\end{array}$ & $<0,00$ I \\
\hline \%Talla: & Según edad Pubetal & $\begin{array}{c}99,91 \pm 4,19 \\
(98)\end{array}$ & $\begin{array}{c}96,31 \pm 4,44 \\
(58)\end{array}$ & $<0,001$ \\
\hline & & N.S. & $<0,001$ & \\
\hline
\end{tabular}

$\overline{\mathrm{A}}=\overline{\mathrm{X}} \pm \mathrm{DE}$

(N) 
escolares de sexo masculino de 13 años en etapa II y IV de vello púbico. Esta fue significativamente menor en escolares en etapa Il si se expresa según $T / E$ y significativamente mayor si se expresa según $T / P$, al compararlos con escolares en etapa IV de vello púbico. En varones en etapa $\mathrm{II}$, la $\mathrm{T} / \mathrm{E}$ es significativamente menor que la $T / P$, en tanto, en varones en etapa $I V$, sucedió lo contrario,

En la Tabla 5 se analiza la estatura de varones de 15 años en etapas II y IV de vello púbico. Al expresar el porcentaje de adecuación estatural según $T / E$, esta es significativamente menor en escolares en etapa $\mathbf{I}$. Si se expresa según $T / P$, ésta es similar en etapa II y IV. Las ade cuaciones estaturales según $T / E$, son significativamente menores a las obtenidas según $T / P$ en ambos grupos de escolares.

\section{DISCUSION}

Nos pareció interesante, conocer las diferencias que existían en la adecuación estatural en escolares de Santiago, según se analizara de

Tabla 3.

Talla en escolares de 13 afios de sexo femenino con diferentes etapas de desarrollo mamario (Tanner)

\begin{tabular}{lcccc} 
& & II & IV & P \\
\hline \% Talla: & Según edad Cronológica & $\begin{array}{c}93,79 \pm 3,76 \mathrm{~A} \\
(15)\end{array}$ & $\begin{array}{c}97,26 \pm 3,98 \\
(223)\end{array}$ & $<0,001$ \\
\hline$\%$ Talla: & Según edad Puberal & $103,91 \pm 4,26$ & $(15)$ & $98,92 \pm 3,96$ \\
& & $<0,001$ & $<23)$ & $<0,001$ \\
\hline
\end{tabular}

$\underline{\mathrm{A}=\overline{\mathrm{X}} \pm \underline{\mathrm{DE}}}$

(N)

Tabla 4 ,

Talla en escolares de 13 años de scxa masculino con diferentes etapas de desarrollo de vello púbico (Tanner)

\begin{tabular}{|c|c|c|c|c|}
\hline & & Il & IV & $\mathrm{P}$ \\
\hline$\%$ I alla: & Según edad Cronológia & $\begin{array}{c}96.73 \pm 4,60^{A} \\
(95)\end{array}$ & $\underset{(59)}{103,11 \pm 3,98}$ & $<0,001$ \\
\hline \multirow[t]{2}{*}{$\%$ Talla: } & Según edad Puberal & $\begin{array}{c}100,38 \pm 4,76 \\
(95)\end{array}$ & $\begin{array}{c}98,03 \pm 3,54 \\
(59)\end{array}$ & $<0,001$ \\
\hline & & $<0,001$ & $<0,001$ & \\
\hline
\end{tabular}

$\frac{\mathrm{A}=\overline{\mathrm{X}} \pm \mathrm{DE}}{(\mathrm{N})}$

Tabla 5.

Talla en escolares de 15 años de sexo masculino con diferentes etapas de desarrollo de vello púbico (Tanner)

\begin{tabular}{lcccc}
\hline & & II & TV & P \\
\hline \%Talla: & Según edad Cronológica & $\begin{array}{c}90,03 \pm 3,27 \mathrm{~A} \\
(10)\end{array}$ & $\begin{array}{c}96,73 \pm 3,90 \\
(107)\end{array}$ & $<0,001$ \\
\hline \%Talla: & Según edad Puberal & $\begin{array}{c}102,04 \pm 3,70 \\
(10)\end{array}$ & $\begin{array}{c}100,50 \pm 4,11 \\
(107)\end{array}$ & N.S. \\
\hline & & $<0,001$ & $<0,001$ & \\
\hline
\end{tabular}

$$
\frac{A=\bar{X}+D E}{(N)}
$$


acuerdo a edad cronológica o a edad de desarrollo puberal en sujetos con eventos puberales, tempranos, normales o tardíos. Esta información permitiria hacer una mejor evaluación estatural para formular un pronóstico más adecuado de estatura final.

En escolares de Santiago de ambos sexos se encontró un mayor promedio estatural en los que inician los eventos puberales a mayor edad. Al analizat la estatura según edad cronológica, ésta es mayor mientras más temprano se inician los eventos. Esto no se traduce en una mayor estatura final, ya que es sabido que mientras más temprano se inicia la pubertad, la estatura final alcanzada es menor, debido a un cierre más temprano de los cartílagos de crecimiento. Esto concuerda con lo descrito por Tanner22 y Frisch ${ }^{10}$ qujenes sen̄alan que la estatura final depende de la edad de inicio del desarrolio puberal.

Al expresar la estatura de estos escolares de acuerdo al grado de desarrollo puberal alcanzado, ésta es menor en los escolares con pubertades más tempranas y mayor en aquellos con pubertades mâs tardias. Esto coincidiría con el hecho que la estimación de pronóstico de talla final es mayor en los sujetos con cierto retraso de la edad ósea al compararlos con sujetos en que existe un cierto grado de adelanto de la maduración ósea ${ }^{14,10,21,22}$

Nuestros resulrados demuestran que en los escolares que presentan los eventos puberales a una edad promedio, la estatura obtenida según edad cronológica ( $T / E$ ) es similar a la pronosticada según desarrollo puberal (T/P), en casos de eventos puberales tempranos o tardios ambos pronósticos son significativamente diferentes. $\mathrm{La}$ información obtenida refuerza además la necesidad de expresar la estatura considerando el grado de desarrollo puberal alcanzado, pues de este modo, puede estimarse en forma más adecuada la calidad de talla final que alcanzará el sujeto, especialmente en adolescentes con presentación temprana o tardía de los eventos puberales.

\section{RESUMEN}

Se estudió las características de la pubertad en un "corte transversal", de 665 escolares de Santiago, de ambos sexos, de 11 a 16 años de edad, de los cuales 394 eran mujeres y 271 varones. Se evaluó lá estatura según edad cronológica $(T / E)$ y según grado de desarrollo puberal alcanzado (T/P) de acuerdo a las escalas de Tanner. Se consideró el desarrollo mamario en las mujeres y el vello púbico en los varones.

Los escolares con pubertad temprana, presentaron un porcentaje de adecuación de $\mathrm{T} / \mathrm{E}$ signi- ficativamente mayor que la $\mathrm{T} / \mathrm{P}$, resuitando con una estatura normal para la edad, pero baja para el grado de desarrollo puberal. Los escolares con pubertad tardía, presentaron una $T / E$ menor que la $T / P$ y su estatura resultó baja para la edad, pero normal para el grado de desarrollo puberal alcanzado. Se concluye la necesidad de conside. rar el grado de desarrollo puberal en la evaluación estatural para un pronóstico más adecuado de la estatura final.

\section{REFERENCIAS}

1. U.S. Department of Health Education and tielfare Public Health Service. NCHS Growth Curves for Children: Birth 18 years. United States. D.H.E.W. 1977. Vital and Health Statistics series 11, number $165,1977$.

2. Smith, $D$.: Measurents, methods and standards, In Growth and its Disorders: Basics and Standards, Approach and Classifications, Growth Deficiency Disorders, Growth Excess Disorders, Obesity. Smith, D. (Ed.) W.B. Saunders Company, Philadelphia, London, Toronto, 1977 , pp. 18-61.

3. Sims, M. Cortinez, A., Schneider, R., Rivas, 1 . Desarrollo puberal y estado nutritivo. Rev. Chil. Pediatr. 41: 291, 1970.

4. Sims, M., Rivos, I., Schneider, R., Cortínez, A.: Desarfollo puberal femenino-menarquia. Kev. Chil. Pediatr. 41: $202,1970$.

5. Vatenzuela, C.: Adelanto de la pubertad en escolares chilenos respecto a escolares europeos medido por la velocidad de crecimiento estatural en un estudio transversal. Pediatría (Santiago) 20; 74, 1977.

6. Valenzuela, $C$, Avendoño, A, Patri. A, Sepúlueda, $H$.: Tamanio testicular en escolates. Rev. Chil. Pediatr. 54: 240, 1983.

7. Pereiro, $C$, Mendoza, M. Femández, $M$.: Desarrollo puberal en escolares de Merida. Acta Cient. Venez. 33: 161, 1982.

8. Morshall, $W$, Tanner, $J_{1}$ : Variations in the Pattern of Puberal changes in boys. Arch, Dis, Child. 45: 13,1970 .

9. Marshall, W. Tanner, J.: Variations in Pattern of Puberal changes in girls. Arch. Dis. Child. 44: 291, 1970.

10. Frisch, R., Reyelle, $R$.: The Height and Weight of girls and boys at the time of initiation of the adolescent growth spurt in height and weight and the relationship to menarche. Hum. Biol. 43: 140, 1971.

11. Frisch, R., Revelle, R.: The Height and Weight of Adolescent boys and girls at the time of peak velocity of growth in height and weight: Longitudinal data. Hum. Biol, 41: 536, 1969.

12. Frisch, R., Nagel, S.: Prediction of Adult height of girls from age of menarche and height at menarche. J. Pediatr. 85: 838, 1974.

13. Bayley, $N_{\text {.: }}$ Tables for predicting adult height from skeletal age and present height. J. Pediatr. 28:49, 1946.

14. Bayley, $N$. Pinneat, $S$.: Tables for predicting adult loight from skeletal age: Revised for use with Greulich-Pyle hand standars. J. Pediatr. 40: 23, 1952. 
15. Avendaño, A., Valenzuela, C., Costa, R., y cols.: Estudio alimentario de escolares con alta $\mathrm{y}$ baja estatura según maduración ósea. Area Norte de Santiago, 1974. Pediatría (Santiago) 19: 7, 1976.

16. Onot, T., Numan-Cebeci, E.: Sesamoid bones of the hand. Relationships to growth skeletal and sexual development in girls. Hum. Biol. 48: 659, 1976.

17. Roche, A., Wainer, H, Thissen, D.: The RWT Method for the Prediction of Adults Stature. Pediatrics 56: 1026, 1975 .

18. Posf, E.. Richmann, R.: A condensed table for predicting adult stature. J. Pediat. 98: 440, 1981.

19. Tanner, $f$.: Puberty. In: Foetus into Man Physical growth from Conception to Maturity. Tanner, J.
(Ed.) Harward. University Press. Cambrige, Massachusetts, 1978. pp. 61-77.

20. Morsholl, W.: Puberty. In Human Growth 2 Post sutal Growth. Falkner, F, and Tamer, J, (Fds.). Plenum Press. New York and London. 1978, pp. 141 - 181 .

21. Tanner, J.: Developmental Age, and the problems of early and late Maturers. In Foetus into man. Physical Growth from Conception to Maturity. Tanner, J. Ed. Harward. University Press Cambridge, Massachusetts 1978, pp. 79-86.

22. Burrows, R.: Exceso de estatura. En: crecimiento y Desarrollo Normal y Patológico. Muzzo, S, (Ed.) Instituto Profesional de Chilán, 1985, pp. 28-31. 